\title{
Dynamic Optimization of a Polymer Flooding Process Based on Implicit Discrete Maximum Principle
}

\author{
Yang Lei, ${ }^{1}$ Shurong Li, ${ }^{1}$ Xiaodong Zhang, ${ }^{1}$ \\ Qiang Zhang, ${ }^{1}$ and Lanlei Guo ${ }^{2}$ \\ ${ }^{1}$ College of Information and Control Engineering, China University of Petroleum, East China, \\ Qingdao 266580, China \\ ${ }^{2}$ Research Institute of Geological Science, Sinopec Shengli Oilfield Company, \\ Dongying 257015, China
}

Correspondence should be addressed to Yang Lei, yutian_hdpu2003@163.com

Received 30 May 2012; Accepted 29 June 2012

Academic Editor: Piermarco Cannarsa

Copyright (C) 2012 Yang Lei et al. This is an open access article distributed under the Creative Commons Attribution License, which permits unrestricted use, distribution, and reproduction in any medium, provided the original work is properly cited.

\begin{abstract}
Polymer flooding is one of the most important technologies for enhanced oil recovery (EOR). In this paper, an optimal control model of distributed parameter systems (DPSs) for polymer injection strategies is established, which involves the performance index as maximum of the profit, the governing equations as the fluid flow equations of polymer flooding, and some inequality constraints as polymer concentration and injection amount limitation. The optimal control model is discretized by full implicit finite-difference method. To cope with the discrete optimal control problem (OCP), the necessary conditions for optimality are obtained through application of the calculus of variations and Pontryagin's discrete maximum principle. A modified gradient method with new adjoint construction is proposed for the computation of optimal injection strategies. The numerical results of an example illustrate the effectiveness of the proposed method.
\end{abstract}

\section{Introduction}

It is of increasing necessity to produce oil fields more efficiently and economically because of the ever-increasing demand for petroleum worldwide. Since most of the significant oil fields are mature fields and the number of new discoveries per year is decreasing, the use of EOR processes is becoming more and more imperative. At present, polymer flooding technology is the best method for chemically EOR [1]. It could reduce the water-oil mobility ratio and improve sweep efficiency [2-5]. 
Due to the high cost of chemicals, it is essential to optimize polymer injection strategies to provide the greatest oil recovery at the lowest cost. The optimization procedure involves maximizing the objective function (cumulative oil production or profit) from a polymer flooding reservoir by adjusting the injection concentration. One way of solving this problem is direct optimization with the reservoir simulator. Numerical models are used to evaluate the complex interactions of variables affecting development decisions, such as reservoir and fluid properties and economic factors. Even with these models, the current practice is still the conventional trial and error approach. In each trial, the polymer concentration of an injection well is selected based on the intuition of the reservoir engineer. This one-well-at-atime approach may lead to suboptimal decisions because engineering and geologic variables affecting reservoir performance are often nonlinearly correlated. And the problem definitely compounds when multiple producers and injectors are involved in a field development case. The use of the optimal control method offers a way out.

The optimal control method has been researched in EOR techniques in recent years. Ramirez et al. [6] firstly applied the theory of optimal control to determine the best possible injection strategies for EOR processes. Their study was motivated by the high operation costs associated with EOR projects. The objective of their study was to develop an optimization method to minimize injection costs while maximizing the amount of oil recovered. The performance of their algorithm was subsequently examined for surfactant injection as an EOR process in a one-dimensional core flooding problem [7]. The control for the process was the surfactant concentration of the injected fluid. They observed a significant improvement in the ratio of the value of the oil recovered to the cost of the surfactant injected from 1.5 to about 3.4. Optimal control was also applied to steam flooding by Liu et al. [8]. They developed an approach using optimal control theory to determine operating strategies to maximize the economic attractiveness of steam flooding process. Their objective was to maximize a performance index which is defined as the difference between oil revenue and the cost of injected steam. Their optimization method also obtained significant improvement under optimal operation. Ye et al. [9] were involved in the study of optimal control of gascycling in condensate reservoirs. It was shown that both the oil recovery and the total profit of a condensate reservoir can be enhanced obviously through optimization of gas production rate, gas injection rate, and the mole fractions of each component in injection gas. Brouwer and Jansen [10], Sarma et al. [11], Zhang et al. [12], and Jansen [13] used the optimal control theory as an optimization algorithm for adjusting the valve setting in smart wells of water flooding. The water flooding scheme that maximized the profit was numerically obtained by combining reservoir simulation with control theory practices of implicit differentiation. They were able to achieve improved sweep efficiency and delayed water breakthrough by dynamic control of the valve setting.

For the previous work on optimal control of polymer flooding, Guo et al. [14] and Lei et al. [15] applied the iterative dynamic programming algorithm to solve the OCP of polymer flooding. However, the optimal control model used in their study is so simple that it is not adapt for practical oilfield development. As a result of the complicated nature of reservoir models with nonlinear constraints, it is very tedious and troublesome to cope with a large number of grid points for the state variables and control variables. To avoid these difficulties, Li et al. [16] and Lei et al. [17, 18] used the genetic algorithms to determine the optimal injection strategies of polymer flooding and the reservoir model equations were treated as a "black box." The genetic algorithms are capable of finding the global optimum on theoretical sense, but as Sarma et al. [11] point out, they require a tens or hundreds of thousand reservoir 
simulation runs of very large model and are not able to guarantee monotonic maximization of the objective function.

In this paper, an optimal control model of DPS for polymer flooding is established which maximizes the profit by adjusting the injection concentration. Then, the determination of polymer injection strategies turns to solve this OCP of DPS. The model is discretized by the full implicit finite difference method. Necessary conditions for optimality are obtained by Pontryagin's discrete maximum principle. A new gradient-based numerical algorithm which makes it relatively easy to create adjoint equations is presented for solving the OCP. Finally, an example of polymer flooding project involving a heterogeneous reservoir case is investigated and the results show the efficiency of the proposed method.

\section{Mathematical Formulation of Optimal Control}

\subsection{Performance Index}

Let $\Omega \in R^{2}$ denote the domain of reservoir with boundary $\partial \Omega$, let $n$ be the unit outward normal on $\partial \Omega$, and let $(x, y) \in \Omega$ be the coordinate of a point in the reservoir. Given a fixed final time $t_{f}$, we set $\Psi=\Omega \times\left[0, t_{f}\right], \Gamma=\partial \Omega \times\left[0, t_{f}\right]$, and suppose that there exist $N_{w}$ injection wells and $N_{o}$ production wells in the oilfield. The injection and production wells are located at $L_{w}=\left\{\left(x_{w i}, y_{w i}\right) \mid i=1,2, \ldots, N_{w}\right\}$ and $L_{o}=\left\{\left(x_{o j}, y_{o j}\right) \mid j=1,2, \ldots, N_{o}\right\}$, respectively. This descriptive statement of the cost functional must be translated into a mathematical form to use quantitative optimization techniques. The oil value can be formulated as

$$
\int_{0}^{t_{f}} \iint_{\Omega} \xi_{o}\left(1-f_{w}\right) q_{\text {out }} d \sigma d t
$$

where $\xi_{o}$ is the cost of oil per unit mass $\left(10^{4} \$ / \mathrm{m}^{3}\right), f_{w}(x, y, t)$ is the fractional flow of water, and $q_{\text {out }}(x, y, t)$ is the flow velocity of production fluid (m/day). We define $q_{\text {out }}(x, y, t) \geq 0$ at $(x, y) \in L_{o}$ and $q_{\text {out }}(x, y, t) \equiv 0$ at $(x, y) \notin L_{o}$.

The polymer cost is expressed mathematically as

$$
\int_{0}^{t_{f}} \iint_{\Omega} \xi_{p} q_{\text {in }} c_{\text {pin }} d \sigma d t
$$

where $\xi_{p}$ is the cost of oil per unit volume $\left(10^{4} \$ / \mathrm{m}^{3}\right), c_{\text {pin }}(x, y, t)$ is the polymer concentration of the injection fluid (g/L), and $q_{\text {in }}(x, y, t)$ is the flow velocity of injection fluid (m/day). We define $q_{\text {in }}(x, y, t) \geq 0$ at $(x, y) \in L_{w}$ and $q_{\text {in }}(x, y, t) \equiv 0$ at $(x, y) \notin L_{w}$.

The objective functional is, therefore,

$$
\max J=\int_{0}^{t_{f}} \iint_{\Omega}\left[\xi_{o}\left(1-f_{w}\right) q_{\text {out }}-\xi_{p} q_{\text {in }} c_{\text {pin }}\right] d \sigma d t .
$$

\subsection{Governing Equations}

The maximization of the cost functional $J$ given by (2.3) is not totally free but is constrained by the system process dynamics. The governing equations of the polymer flooding process 
must, therefore, be developed to describe the flow of both the aqueous and oil phases through the porous media of a reservoir formation. The equations used in this paper allow for the adsorption of polymer onto the solid matrix in addition to the convective and dispersive mechanisms of mass transfer. Let $p(x, y, t), S_{w}(x, y, t)$, and $c_{p}(x, y, t)$ denote the pressure, water saturation, and polymer concentration of the oil reservoir, respectively, at a point $(x, y) \in \Omega$ and a time $t \in\left[0, t_{f}\right]$, then $p(x, y, t), S_{w}(x, y, t)$, and $c_{p}(x, y, t)$ satisfy the following partial differential equations (PDEs).

(1) The flow equation for oil phase:

$$
\frac{\partial}{\partial x}\left(k_{p} r_{o} \frac{\partial p}{\partial x}\right)+\frac{\partial}{\partial y}\left(k_{p} r_{o} \frac{\partial p}{\partial y}\right)-\left(1-f_{w}\right) q_{\text {out }}=h \frac{\partial}{\partial t}\left[\frac{\phi\left(1-S_{w}\right)}{B_{o}}\right], \quad(x, y, t) \in \Psi .
$$

(2) The flow equation for water phase:

$$
\frac{\partial}{\partial x}\left(k_{p} r_{w} \frac{\partial p}{\partial x}\right)+\frac{\partial}{\partial y}\left(k_{p} r_{w} \frac{\partial p}{\partial y}\right)+q_{\text {in }}-f_{w} q_{\mathrm{out}}=h \frac{\partial}{\partial t}\left(\frac{\phi S_{w}}{B_{w}}\right), \quad(x, y, t) \in \Psi .
$$

(3) The flow equation for polymer component:

$$
\begin{gathered}
\frac{\partial}{\partial x}\left(k_{d} r_{d} \frac{\partial c_{p}}{\partial x}\right)+\frac{\partial}{\partial x}\left(k_{p} r_{c} \frac{\partial p}{\partial x}\right)+\frac{\partial}{\partial y}\left(k_{d} r_{d} \frac{\partial c_{p}}{\partial y}\right)+\frac{\partial}{\partial y}\left(k_{p} r_{c} \frac{\partial p}{\partial y}\right)+q_{\text {in }} c_{\text {pin }}-f_{w} q_{\text {out }} c_{p} \\
=h \frac{\partial}{\partial t}\left[\frac{\phi_{p} S_{w} c_{p}}{B_{w}}+\rho_{r}(1-\phi) C_{\mathrm{rp}}\right], \quad(x, y, t) \in \Psi .
\end{gathered}
$$

(4) The boundary conditions and initial conditions:

$$
\begin{gathered}
\left.\frac{\partial p}{\partial n}\right|_{\partial \Omega}=0,\left.\quad \frac{\partial S_{w}}{\partial n}\right|_{\partial \Omega}=0,\left.\quad \frac{\partial c_{p}}{\partial n}\right|_{\partial \Omega}=0, \quad(x, y, t) \in \Gamma \\
p(x, y, 0)=p^{0}(x, y), \quad S_{w}(x, y, 0)=S_{w}^{0}(x, y), \quad c_{p}(x, y, 0)=c_{p}^{0}(x, y), \quad(x, y) \in \Omega .
\end{gathered}
$$

where the corresponding parameters are defined as

$$
\begin{gathered}
k_{p}=K h, \quad k_{d}=D h, \\
r_{o}=\frac{k_{r o}}{B_{o} \mu_{o}}, \quad r_{w}=\frac{k_{r w}}{B_{w} R_{k} \mu_{w}}, \quad r_{c}=\frac{k_{r w} c_{p}}{B_{w} R_{k} \mu_{p}}, \quad r_{d}=\frac{\phi_{p} S_{w}}{B_{w}} .
\end{gathered}
$$

The constant coefficient $K(x, y)$ is the absolute permeability $\left(\mu \mathrm{m}^{2}\right), D(x, y)$ is the diffusion coefficient of polymer $\left(\mathrm{m}^{2} / \mathrm{s}\right), h(x, y)$ is the thickness of the reservoir bed $(\mathrm{m}), \rho_{r}$ is the rock density $\left(\mathrm{kg} / \mathrm{m}^{3}\right)$, and $\mu_{o}$ is the oil viscosity $(\mathrm{mPa} \cdot \mathrm{s})$. 
The oil volume factor $B_{o}$, the water volume factor $B_{w}$, the rock porosity $\phi$, and the effective porosity to polymer $\phi_{p}$ are expressed as functions of the reservoir pressure $p$ :

$$
\begin{gathered}
B_{o}=\frac{B_{o r}}{\left[1+C_{o}\left(p-p_{r}\right)\right]}, \\
B_{w}=\frac{B_{w r}}{\left[1+C_{w}\left(p-p_{r}\right)\right]}, \\
\phi=\phi_{r}\left[1+C_{R}\left(p-p_{r}\right)\right], \\
\phi_{p}=f_{a} \phi,
\end{gathered}
$$

where $p_{r}$ is the reference pressure (MPa), $\phi_{r}, B_{o r}$, and $B_{w r}$ denote the porosity, the oil and water volume factor under the condition of the reference pressure, respectively, $f_{a}$ is the effective pore volume coefficient, $C_{o}, C_{w}$, and $C_{R}$ denote the compressibility factors of oil, water, and rock, respectively.

Functions relating values of the oil and water relative permeabilities $k_{r o}$ and $k_{r w}$ to the water saturation $S_{w}$ are

$$
\begin{aligned}
& k_{r w}=k_{r w r o}\left(\frac{S_{w}-S_{w c}}{1-S_{w c}-S_{o r}}\right)^{n_{w}} \\
& k_{r o}=k_{\text {rocw }}\left(\frac{1-S_{w}-S_{o r}}{1-S_{w c}-S_{o r}}\right)^{n_{o}}
\end{aligned}
$$

where $k_{r w r o}$ is the oil relative permeability at the irreducible water saturation $S_{w c}, k_{r w c w}$ is the water relative permeability at the residual oil saturation $S_{o r}, n_{w}$, and $n_{o}$ are constant coefficients.

The polymer solution viscosity $\mu_{p}(\mathrm{mPa} \cdot \mathrm{s})$, the permeability reduction factor $R_{k}$, and the amount adsorbed per unit mass of the rock $C_{r p}(\mathrm{mg} / \mathrm{g})$ which depend on the polymer concentration $c_{p}$ are given by

$$
\begin{gathered}
\mu_{p}=\mu_{w}\left[1+\left(a p_{1} c_{p}+a p_{2} c_{p}^{2}+a p_{p}^{3}\right)\right] \\
R_{k}=1+\frac{\left(R_{k \max }-1\right) \cdot b_{r k} \cdot c_{p}}{1+b_{r k} \cdot c_{p}}, \\
C_{r p}=\frac{a c_{p}}{1+b c_{p}},
\end{gathered}
$$

where $\mu_{w}$ is the viscosity of the aqueous phase with no polymer (mPa.s), ap $1, a p_{2}, a p_{3}, R_{k \max }$, $b_{r k}, a\left(\mathrm{~cm}^{3} / \mathrm{g}\right)$, and $b(\mathrm{~L} / \mathrm{g})$ are constant coefficients.

The fractional flow of water $f_{w}$ is given by

$$
f_{w}=\frac{r_{w}}{r_{o}+r_{w}}
$$




\subsection{Constraints}

Since the polymer injection amount is limited, and the negative and overhigh injection concentrations are not allowed, the constraints in polymer flooding are expressed mathematically as follows.

(1) The polymer injection amount constraint:

$$
\int_{0}^{t_{f}} \iint_{\Omega} q_{\text {in }} c_{\text {pin }} d \sigma d t \leq m_{p \max }
$$

(2) The injection polymer concentration constraint:

$$
0 \leq c_{\text {pin }} \leq c_{\max }
$$

where $m_{p \max }$ is the maximum polymer injection amount and $c_{\max }$ is the maximum injection polymer concentration.

\section{Full Implicit Finite Difference Method}

The governing equations given by (2.4)-(2.8) are nonlinear PDEs. Several finite difference approximations for the numerical simulation of such DPS are possible. We adopt a fullimplicit finite-difference scheme for the calculation of the governing equations. The scheme is described below.

For two space variables, we now consider the grid system with which we divide up the reservoir region in the $x$-y plane. The integer $i\left(i=1,2, \ldots, n_{x}\right)$ is used as the index in the $x$-direction, and the integer $j\left(j=1,2, \ldots, n_{y}\right)$ for the index in the $y$-direction. Thus, $x_{i}$ is the $i$ th value of $x$, and $y_{j}$ is the $j$ th value of $y$. Double indexing is used to identify functions within the two-dimensional region. Let $\mathbf{u}(x, y, t)=\left[p, S_{w}, c_{p}\right]^{T}$ denote the system state vector. The discrete state vector in grid point $\left(x_{i}, y_{j}\right)$ is described by

$$
\mathbf{u}_{i, j}=\mathbf{u}\left(x_{i}, y_{j}\right)
$$

The reservoir domain is divided into $n_{x} \times n_{y}$ blocks, and the point $\left(x_{i}, y_{j}\right)$ is considered to be at the center of block $(i, j)$. There are $n_{x}$ blocks in the $x$-direction and $n_{y}$ blocks in the $y$-direction. Further details concerning this grid are given in Figure 1. We identify the coordinate $x_{i-(1 / 2)}$ with the left side of the block $(i, j)$, and $x_{i+(1 / 2)}$ with the right side of the block. Similarly, $y_{j-(1 / 2)}$ is identified with the bottom of the block, and $y_{j+(1 / 2)}$ with the top. 


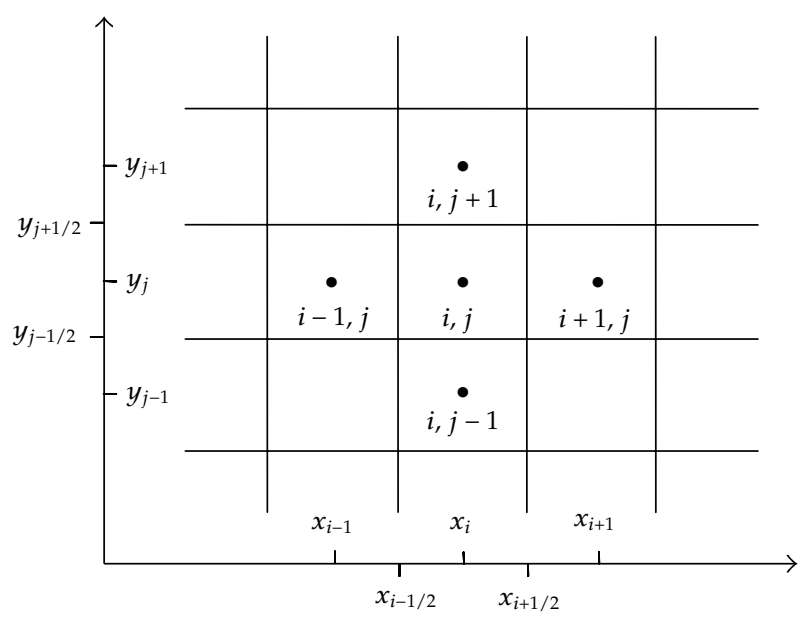

Figure 1: Details of block-centred grid.

Using the predefined grid system, the derivatives in (2.4)-(2.6) are replaced by finite differences. Three formulas are useful in this context:

$$
\begin{gathered}
\frac{\partial \mathbf{u}}{\partial t}=\frac{\mathbf{u}^{n+1}-\mathbf{u}^{n}}{\Delta t^{n}} \\
\frac{\partial \mathbf{u}}{\partial x}=\frac{\mathbf{u}_{i+(1 / 2), j}-\mathbf{u}_{i-(1 / 2), j}}{\Delta x}, \\
\frac{\partial \mathbf{u}}{\partial y}=\frac{\mathbf{u}_{i, j+(1 / 2)}-\mathbf{u}_{i, j-(1 / 2)}}{\Delta y}
\end{gathered}
$$

where $n=0,1, \ldots, N-1$ is the index of reservoir simulation time step, $N$ is the number of simulation time steps, $\Delta t^{n}$ represents the size of the $n$th time step in days, $\mathbf{u}^{n+1}$ represents the state vector at time $t^{n+1}, t^{n+1}$ is the total simulation time in days at the end of the $n$th time step $\left(t^{N}=t_{f}\right)$, and $\Delta x$ and $\Delta y(\mathrm{~m})$ denote the space step lengths in the $x$-direction and $y$-direction, respectively.

Apply the formulas (3.2) to discretize the governing equations (2.4)-(2.6), and multiply the grid area $\Delta x \Delta y\left(\mathrm{~m}^{2}\right)$ to the two sides of the equations. For the full implicit finite difference method, the second spatial derivative items in (2.4)-(2.6) are evaluated at time $t^{n+1}$ instead of at $t^{n}$. Then, the full-implicit finite-difference equations of the polymer flooding model has the general form:

$$
\mathbf{g}^{n}=\mathbf{F}^{n+1}\left(\tilde{\mathbf{u}}^{n+1}\right)+\mathbf{W}^{n+1}\left(\tilde{\mathbf{u}}^{n+1}, \mathbf{v}^{n}\right)-\left[\mathbf{A}^{n+1}\left(\tilde{\mathbf{u}}^{n+1}\right)-\mathbf{A}^{n}\left(\tilde{\mathbf{u}}^{n}\right)\right]=\mathbf{0},
$$

where $\mathbf{g}=\left[\mathbf{g}_{1,1}^{T}, \ldots, \mathbf{g}_{i, j}^{T}, \ldots, \mathbf{g}_{n_{x}, n_{y}}^{T}\right]^{T} \in R^{n_{x} \times n_{y} \times 3}$ is the discrete governing equations of polymer flooding model, $\tilde{\mathbf{u}}=\left[\mathbf{u}_{1,1}^{T}, \ldots, \mathbf{u}_{i, j}^{T}, \ldots, \mathbf{u}_{n_{x}, n_{y}}^{T}\right]^{T} \in R^{n_{x} \times n_{y} \times 3}$ is the discrete state vector, $\mathbf{v}=$ $\left[v_{i, j} \mid v_{i, j}=c_{\text {pin } i, j},(i, j) \in \kappa_{w}\right]^{T} \in R^{N_{w}}$ is the control vector, $\kappa_{w}$ is the set of grid coordinates where injection wells located, $\mathbf{F}$ refers to the flux terms, $\mathbf{W}$ refers to the source terms, and $\mathbf{A}$ refers to the accumulation terms. 
The discrete governing equations $\mathbf{g}_{i, j}=\left[g_{o i, j}, g_{w i, j}, g_{c i, j}\right]^{T}$ for the grid point $(i, j)$ at time $t^{n}$ are given by

$$
\begin{gathered}
g_{o i, j}^{n}=F_{o i, j}^{n+1}+W_{o i, j}^{n+1}-A_{o i, j}^{n+1}+A_{o i, j}^{n}=0, \\
g_{w i, j}^{n}=F_{w i, j}^{n+1}+W_{w i, j}^{n+1}-A_{w i, j}^{n+1}+A_{w i, j}^{n}=0, \\
g_{c i, j}^{n}=F_{c i, j}^{n+1}+W_{c i, j}^{n+1}-A_{c i, j}^{n+1}+A_{c i, j}^{n}=0 .
\end{gathered}
$$

Equations (3.4) are discrete flow equations for oil phase, water phase, and polymer component, respectively. The full-implicit finite-difference schemes for the flux terms $\mathbf{F}_{i, j}=$ $\left[F_{o i, j}, F_{w i, j}, F_{c i, j}\right]^{T}$ are given by

$$
\begin{aligned}
& F_{o i, j}^{n+1}=\Delta y\left[\frac{k_{p_{i+(1 / 2), j}} r_{o_{i+(1 / 2), j}}^{n+1}}{\Delta x}\left(p_{i+1, j}^{n+1}-p_{i, j}^{n+1}\right)+\frac{k_{p_{i-(1 / 2), j}} r_{o_{i-(1 / 2), j}}^{n+1}}{\Delta x}\left(p_{i-1, j}^{n+1}-p_{i, j}^{n+1}\right)\right] \\
& +\Delta x\left[\frac{k_{p_{i, j+(1 / 2)}} r_{o_{i, j+(1 / 2)}}^{n+1}}{\Delta y}\left(p_{i, j+1}^{n+1}-p_{i, j}^{n+1}\right)+\frac{k_{p_{i, j-(1 / 2)}} r_{o_{i, j}(1 / 2)}^{n+1}}{\Delta y}\left(p_{i, j-1}^{n+1}-p_{i, j}^{n+1}\right)\right] \text {, } \\
& F_{w i, j}^{n+1}=\Delta y\left[\frac{k_{p_{i+(1 / 2), j}} r_{w_{i+(1 / 2), j}}^{n+1}}{\Delta x}\left(p_{i+1, j}^{n+1}-p_{i, j}^{n+1}\right)+\frac{k_{p_{i-(1 / 2), j}} r_{w_{i-(1 / 2), j}}^{n+1}}{\Delta x}\left(p_{i-1, j}^{n+1}-p_{i, j}^{n+1}\right)\right] \\
& +\Delta x\left[\frac{k_{p_{i, j+(1 / 2)}} r_{w_{i, j+(1 / 2)}}^{n+1}}{\Delta y}\left(p_{i, j+1}^{n+1}-p_{i, j}^{n+1}\right)+\frac{k_{p_{i, j-(1 / 2)}} r_{w_{i, j-(1 / 2)}^{n+1}}}{\Delta y}\left(p_{i, j-1}^{n+1}-p_{i, j}^{n+1}\right)\right],
\end{aligned}
$$

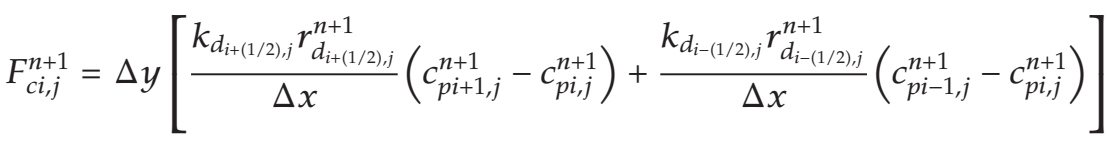

$$
\begin{aligned}
& +\Delta x\left[\frac{k_{d_{i, j+(1 / 2)}} r_{d_{i, j+(1 / 2)}^{n+1}}^{n+1}}{\Delta y}\left(c_{p i, j+1}^{n+1}-c_{p i, j}^{n+1}\right)+\frac{k_{d_{i, j-(1 / 2)}} r_{d_{i, j-(1 / 2)}^{n+1}}}{\Delta y}\left(c_{p i, j-1}^{n+1}-c_{p i, j}^{n+1}\right)\right] \\
& +\Delta y\left[\frac{k_{p_{i+(1 / 2), j}} r_{c_{i+(1 / 2), j}}^{n+1}}{\Delta x}\left(p_{i+1, j}^{n+1}-p_{i, j}^{n+1}\right)+\frac{k_{p_{i-(1 / 2), j}} r_{c_{i-(1 / 2), j}}^{n+1}}{\Delta x}\left(p_{i-1, j}^{n+1}-p_{i, j}^{n+1}\right)\right] \\
& +\Delta x\left[\frac{k_{p_{i, j+(1 / 2)}} r_{c_{i, j+(1 / 2)}^{n+1}}^{n+1}}{\Delta y}\left(p_{i, j+1}^{n+1}-p_{i, j}^{n+1}\right)+\frac{k_{p_{i, j-(1 / 2)}} r_{c_{i, j-(1 / 2)}}^{n+1}}{\Delta y}\left(p_{i, j-1}^{n+1}-p_{i, j}^{n+1}\right)\right] \text {. }
\end{aligned}
$$

The discrete source terms $\mathbf{W}_{i, j}=\left[W_{o i, j}, W_{w i, j}, W_{c i, j}\right]^{T}$ are expressed as

$$
\begin{gathered}
W_{o i, j}^{n+1}=-\left(1-f_{w i, j}^{n+1}\right) Q_{\text {out } i, j}^{n} \\
W_{w i, j}^{n+1}=Q_{\text {in } i, j}^{n}-f_{w i, j}^{n+1} Q_{\text {out } i, j}^{n}, \\
W_{c i, j}^{n+1}=Q_{\text {in } i, j}^{n} c_{\text {pin } i, j}^{n}-f_{w i, j}^{n+1} Q_{\text {out } i, j}^{n} c_{p i, j}^{n+1},
\end{gathered}
$$


where $Q_{\text {out }}$ is the fluid production rate $\left(Q_{\text {out }}=\Delta x \Delta y q_{\text {out }}, \mathrm{m}^{3} / \mathrm{d}, Q_{\text {out } i, j} \geq 0\right.$ at $(i, j) \in \kappa_{o}$, and $Q_{\text {out } i, j} \equiv 0$ at $\left.(i, j) \notin \kappa_{o}\right), \kappa_{o}$ is the set of grid coordinates where production wells located, $Q_{\text {in }}$ is the fluid injection rate $\left(Q_{\text {in }}=\Delta x \Delta y q_{\text {in }}, \mathrm{m}^{3} / \mathrm{d}, Q_{\text {in } i, j} \geq 0\right.$ at $(i, j) \in \kappa_{w}$, and $Q_{\text {in } i, j} \equiv 0$ at $\left.(i, j) \notin \mathcal{K}_{w}\right)$. The full-implicit finite-difference schemes for the accumulation terms $\mathbf{A}_{i, j}=$ $\left[A_{o i, j}, A_{w i, j}, A_{c i, j}\right]^{T}$ are

$$
\begin{gathered}
A_{o i, j}^{n+1}=\frac{V_{i, j}}{\Delta t^{n}} \frac{\phi_{i, j}^{n+1}\left(1-S_{w i, j}^{n+1}\right)}{B_{o i, j}^{n+1}}, \quad A_{o i, j}^{n}=\frac{V_{i, j}}{\Delta t^{n}} \frac{\phi_{i, j}^{n}\left(1-S_{w i, j}^{n}\right)}{B_{o i, j}^{n}}, \\
A_{w i, j}^{n+1}=\frac{V_{i, j}}{\Delta t^{n}} \frac{\phi_{i, j}^{n+1} S_{w i, j}^{n+1}}{B_{w i, j}^{n+1}}, \quad A_{w i, j}^{n}=\frac{V_{i, j}}{\Delta t^{n}} \frac{\phi_{i, j}^{n} S_{w i, j}^{n}}{B_{w i, j}^{n}}, \\
A_{c i, j}^{n+1}=\frac{V_{i, j}}{\Delta t^{n}}\left[\frac{\phi_{p i, j}^{n+1} S_{w i, j}^{n+1} c_{p i, j}^{n+1}}{B_{w i, j}^{n+1}}+\rho_{r}\left(1-\phi_{i, j}^{n+1}\right) C_{r p i, j}^{n+1}\right], \\
A_{c i, j}^{n}=\frac{V_{i, j}}{\Delta t^{n}}\left[\frac{\phi_{p i, j}^{n} S_{w i, j}^{n} c_{p i, j}^{n}}{B_{w i, j}^{n}}-\rho_{r}\left(1-\phi_{i, j}^{n}\right) C_{r p i, j}^{n}\right],
\end{gathered}
$$

where $V$ is the grid volume $\left(V_{i, j}=h_{i, j} \Delta x \Delta y, \mathrm{~m}^{3}\right)$.

The boundary condition (2.7) is equivalent to

$$
\frac{\partial \mathbf{u}}{\partial x}=0, \quad \frac{\partial \mathbf{u}}{\partial y}=0, \quad \forall(x, y, t) \in \partial \Omega \times\left[0, t_{f}\right]
$$

Therefore, by using finite difference and (3.8), we have

$$
\begin{aligned}
& \mathbf{u}_{i, 0}=\mathbf{u}_{i, 1}, \quad \mathbf{u}_{i, n_{y}+1}=\mathbf{u}_{i, n_{y}}, \quad i=1,2, \ldots, n_{x}, \\
& \mathbf{u}_{0, j}=\mathbf{u}_{1, j}, \quad \mathbf{u}_{n_{x}+1, j}=\mathbf{u}_{n_{x}, j}, \quad j=1,2, \ldots, n_{y} .
\end{aligned}
$$

The initial condition (2.8) is discretized as

$$
\mathbf{u}_{i, j}^{0}=\mathbf{u}\left(x_{i}, y_{j}, 0\right), \quad i=1, \ldots, n_{x}, j=1, \ldots, n_{y} .
$$

In solving the governing equations (2.4)-(2.8) by the full-implicit finite-difference methods, a problem that was originally described by PDEs defined over continuous time and spatial domains is transformed to problem that is described by a set of nonlinear discrete algebraic equations that are implicit form. 


\section{Necessary Conditions of OCP for Polymer Flooding}

By using the full-implicit finite-difference method, the optimal control model of polymer flooding is discretized as the general form:

$$
\begin{gathered}
\max J=\sum_{n=0}^{N-1} J^{n}=\sum_{n=0}^{N-1} \Delta t^{n}\left[\xi_{0} \sum_{(i, j) \in \kappa_{o}}\left(1-f_{w i, j}^{n+1}\right) Q_{\text {out } i, j}^{n}-\xi_{p} \sum_{(i, j) \in \kappa_{w}} c_{\text {pini,j }}^{n} Q_{\text {in } i, j}^{n}\right], \\
\text { s.t. } \quad \mathbf{g}^{n}\left(\tilde{\mathbf{u}}^{n+1}, \widetilde{\mathbf{u}}^{n}, \mathbf{v}^{n}, n\right)=\mathbf{0}, \quad n=0, \ldots, N-1, \\
\left.\tilde{\mathbf{u}}^{n}\right|_{n=0}=\widetilde{\mathbf{u}}^{0}, \\
\sum_{n=0}^{N-1} \Delta t^{n} \sum_{(i, j) \in \kappa_{w}} c_{\text {pin } i, j}^{n} Q_{\text {in } i, j}^{n} \leq m_{p \max }, \\
\quad 0 \leq \mathbf{v}^{n} \leq \mathbf{v}_{\max }
\end{gathered}
$$

where (4.1) is the discrete performance index and (4.4) is the discrete constraint of polymer injection amount.

We desire to find a set of necessary conditions for the state vector, $\widetilde{\mathbf{u}}$, and the control, $\mathbf{v}$, to be extremals of the functional $J$ (4.1) subject to the governing (4.2)-(4.3) and the constraints (4.4)-(4.5). A convenient way to cope with such a discrete OCP is through the use of Pontryagin's maximum principle. The first step is to form an augmented functional by adjoining the governing equations to the performance index $J$ :

$$
J_{A}=\sum_{n=0}^{N-1}\left[J^{n}\left(\widetilde{\mathbf{u}}^{n+1}, \mathbf{v}^{n}, n\right)+\left(\lambda^{n+1}\right)^{T} \mathbf{g}^{n}\left(\widetilde{\mathbf{u}}^{n+1}, \widetilde{\mathbf{u}}^{n}, \mathbf{v}^{n}, n\right)\right],
$$

where $\boldsymbol{\lambda}=\left[\boldsymbol{\lambda}_{1,1}^{T}, \ldots, \boldsymbol{\lambda}_{i, j}^{T}, \ldots, \boldsymbol{\lambda}_{n_{x}, n_{y}}^{T}\right]^{T}$ is the adjoint vector and $\boldsymbol{\lambda}_{i, j}=\left[\boldsymbol{\lambda}_{o i, j}, \boldsymbol{\lambda}_{w i, j}, \boldsymbol{\lambda}_{c i, j}\right]^{T}$.

We can simplify the augmented performance function of (4.6) by introducing the Hamiltonian, $H$, as

$$
H^{n}\left(\widetilde{\mathbf{u}}^{n+1}, \widetilde{\mathbf{u}}^{n}, \mathbf{v}^{n}, \lambda^{n+1}, n\right)=J^{n}\left(\widetilde{\mathbf{u}}^{n+1}, \mathbf{v}^{n}, n\right)+\left(\lambda^{n+1}\right)^{T} \mathbf{g}^{n}\left(\widetilde{\mathbf{u}}^{n+1}, \widetilde{\mathbf{u}}^{n}, \mathbf{v}^{n}, n\right)
$$

Therefore, the augmented performance function can be written as

$$
J_{A}=\sum_{n=0}^{N-1} H^{n}\left(\tilde{\mathbf{u}}^{n+1}, \tilde{\mathbf{u}}^{n}, \mathbf{v}^{n}, \lambda^{n+1}, n\right)
$$


Following the standard procedure of the calculus of variations, the increment of $J_{A}$, denoted by $\Delta J_{A}$, is formed by introducing variations $\delta \widetilde{\mathbf{u}}^{n+1}, \delta \widetilde{\mathbf{u}}^{n}, \delta \mathbf{v}^{n}$, and $\delta \boldsymbol{\lambda}^{n+1}$ giving

$$
\Delta J_{A}=J_{A}\left(\tilde{\mathbf{u}}^{n+1}+\delta \tilde{\mathbf{u}}^{n+1}, \tilde{\mathbf{u}}^{n}+\delta \tilde{\mathbf{u}}^{n}, \mathbf{v}^{n}+\delta \mathbf{v}^{n}, \lambda^{n+1}+\delta \lambda^{n+1}\right)-J_{A}\left(\tilde{\mathbf{u}}^{n+1}, \tilde{\mathbf{u}}^{n}, \mathbf{v}^{n}, \lambda^{n+1}\right) .
$$

This formulation assumes that the final time, $t_{f}$, is fixed.

Expanding (4.9) in a Taylor series and retaining only the linear terms gives the variation of the functional, $\delta J_{A}$,

$$
\delta J_{A}=\sum_{n=0}^{N-1}\left[\left(\frac{\partial H^{n}}{\partial \widetilde{\mathbf{u}}^{n+1}}\right)^{T} \delta \widetilde{\mathbf{u}}^{n+1}+\left(\frac{\partial H^{n}}{\partial \widetilde{\mathbf{u}}^{n}}\right)^{T} \delta \widetilde{\mathbf{u}}^{n}+\left(\frac{\partial H^{n}}{\partial \mathcal{\lambda}^{n+1}}\right)^{T} \delta \boldsymbol{\lambda}^{n+1}+\left(\frac{\partial H^{n}}{\partial \mathbf{v}^{n}}\right)^{T} \delta \mathbf{v}^{n}\right]
$$

The variations $\delta \widetilde{\mathbf{u}}^{n+1}$ and $\delta \widetilde{\mathbf{u}}^{n}$ are not independent but can be related by a discrete version of the integration by parts as

$$
\sum_{n=0}^{N-1}\left(\frac{\partial H^{n}}{\partial \widetilde{\mathbf{u}}^{n+1}}\right)^{T} \delta \widetilde{\mathbf{u}}^{n+1}=\sum_{n=0}^{N-1}\left(\frac{\partial H^{n-1}}{\partial \widetilde{\mathbf{u}}^{n}}\right)^{T} \delta \widetilde{\mathbf{u}}^{n}-\left(\frac{\partial H^{-1}}{\partial \widetilde{\mathbf{u}}^{0}}\right)^{T} \delta \widetilde{\mathbf{u}}^{0}+\left(\frac{\partial H^{N-1}}{\partial \widetilde{\mathbf{u}}^{N}}\right)^{T} \delta \widetilde{\mathbf{u}}^{N}
$$

By substituting (4.11) into (4.10), the first variation $\delta J_{A}$ becomes

$$
\begin{aligned}
\delta J_{A}= & \sum_{n=0}^{N-1}\left(\frac{\partial H^{n-1}}{\partial \widetilde{\mathbf{u}}^{n}}+\frac{\partial H^{n}}{\partial \widetilde{\mathbf{u}}^{n}}\right)^{T} \delta \widetilde{\mathbf{u}}^{n}-\left(\frac{\partial H^{-1}}{\partial \widetilde{\mathbf{u}}^{0}}\right)^{T} \delta \widetilde{\mathbf{u}}^{0}+\left(\frac{\partial H^{N-1}}{\partial \widetilde{\mathbf{u}}^{N}}\right)^{T} \delta \widetilde{\mathbf{u}}^{N} \\
& +\sum_{n=0}^{N-1}\left(\frac{\partial H^{n}}{\partial \mathcal{\ell}^{n+1}}\right)^{T} \delta \boldsymbol{\lambda}^{n+1}+\sum_{n=0}^{N-1}\left(\frac{\partial H^{n}}{\partial \mathbf{v}^{n}}\right)^{T} \delta \mathbf{v}^{n+1} .
\end{aligned}
$$

The following necessary conditions for optimality are obtained when we apply Pontryagin's discrete maximum principle.

\subsection{Adjoint Equations}

Since the variation $\delta \widetilde{\mathbf{u}}^{n}$ is free and not zero, the coefficient terms involving the $\delta \widetilde{\mathbf{u}}^{n}$ variation in (4.12) are set to zero. This results in the following adjoint equations:

$$
\frac{\partial H^{n-1}}{\partial \widetilde{\mathbf{u}}^{n}}+\frac{\partial H^{n}}{\partial \widetilde{\mathbf{u}}^{n}}=0
$$

Substituting the Hamiltonian (4.7) into (4.13), the adjoint equations reduce for the polymer flooding problem under consideration as given by

$$
\left(\frac{\partial \mathbf{g}^{n-1}}{\partial \widetilde{\mathbf{u}}^{n}}\right)^{T} \mathcal{\lambda}^{n}+\left(\frac{\partial \mathbf{g}^{n}}{\partial \widetilde{\mathbf{u}}^{n}}\right)^{T} \mathcal{\lambda}^{n+1}+\frac{\partial J^{n-1}}{\partial \widetilde{\mathbf{u}}^{n}}=0
$$




\subsection{Governing Equations}

Since the variation $\delta \boldsymbol{\lambda}^{n+1}$ is free, we have

$$
\frac{\partial H^{n}}{\partial \mathcal{\Lambda}^{n+1}}=\mathbf{g}^{n}\left(\widetilde{\mathbf{u}}^{n+1}, \widetilde{\mathbf{u}}^{n}, \mathbf{v}^{n}, n\right)=0
$$

\subsection{Transversality Conditions}

Since the initial state is specified, the variation $\delta \widetilde{\mathbf{u}}^{0}$ of (4.12) is zero. However, the final state is not specified; therefore, the variation $\delta \tilde{\mathbf{u}}^{N}$ is free and nonzero. This means the following relation must be zero:

$$
\frac{\partial H^{N-1}}{\partial \widetilde{\mathbf{u}}^{N}}=0
$$

By substituting the Hamiltonian (4.7) into (4.16), the transversality conditions of adjoint equations for the polymer flooding $\mathrm{OCP}$ are expressed as

$$
\frac{\partial J^{N-1}}{\partial \widetilde{\mathbf{u}}^{N}}+\left(\boldsymbol{\lambda}^{N}\right)^{T} \frac{\partial \mathbf{g}^{N-1}}{\partial \widetilde{\mathbf{u}}^{N}}=0 .
$$

Equation (4.17) shows that the adjoint variables are known at the final time $t^{N}$. Since the state variables are known at the initial time and the adjoint variables are known at the final time, the discrete OCP is a split two-point boundary-value problem.

\subsection{Optimal Control}

With all the previous terms vanishing, the variation of the functional $\delta J_{A}$ becomes

$$
\delta J_{A}=\sum_{n=0}^{N-1}\left[\left(\frac{\partial H^{n}}{\partial \mathbf{v}^{n}}\right)^{T} \delta \mathbf{v}^{n}\right] .
$$

When the state and control regions are not bounded, the variation of the functional must vanish at an extremal (the fundamental theorem of the calculus of variations), therefore,

$$
\frac{\partial H^{n}}{\partial \mathbf{v}^{n}}=\frac{\partial J^{n}}{\partial \mathbf{v}^{n}}+\left(\frac{\partial \mathbf{g}^{n}}{\partial \mathbf{v}^{n}}\right)^{T} \lambda^{n+1}=0
$$

Since there are bounds and amount constraints on the control $\mathbf{v}^{n}$, we use the discrete maximum principle to assert the following necessary conditions for optimality:

$$
H^{n}\left(\widetilde{\mathbf{u}}^{n+1}, \widetilde{\mathbf{u}}^{n}, \lambda^{n+1}, \mathbf{v}^{n^{*}}\right)=\max _{\mathbf{v}^{n}} H^{n}\left(\widetilde{\mathbf{u}}^{n+1}, \widetilde{\mathbf{u}}^{n}, \lambda^{n+1}, \mathbf{v}^{n}\right),
$$

where $\mathbf{v}^{n^{*}}$ denotes the optimal control vector. 


\section{Numerical Solution}

A gradient-based iterative numerical technique is proposed to determine the optimal injection strategies of polymer flooding. The computational procedure is based on adjusting estimates of control vector $\mathbf{v}^{n}$ to improve the value of the objective functional. For a control to be optimal, the necessary condition given by (4.20) must be satisfied. If the control $\mathbf{v}^{n}$ is not optimal, then a correction $\delta \mathbf{v}^{n}$ is determined so that the functional is made lager, that is, $\delta J_{A}>0$. If $\delta \mathbf{v}^{n}$ is selected as

$$
\delta \mathbf{v}^{n}=w\left[\frac{\partial J^{n}}{\partial \mathbf{v}^{n}}+\left(\frac{\partial \mathbf{g}^{n}}{\partial \mathbf{v}^{n}}\right)^{T} \lambda^{n+1}\right]
$$

where $w$ is an arbitrary positive weighting factor, the functional variation becomes

$$
\delta J_{A}=\int_{0}^{t_{f}} \iint_{\Omega} w\left[\frac{\partial J^{n}}{\partial \mathbf{v}^{n}}+\left(\frac{\partial \mathbf{g}^{n}}{\partial \mathbf{v}^{n}}\right)^{T} \lambda^{n+1}\right]^{T}\left[\frac{\partial J^{n}}{\partial \mathbf{v}^{n}}+\left(\frac{\partial \mathbf{g}^{n}}{\partial \mathbf{v}^{n}}\right)^{T} \lambda^{n+1}\right] d \sigma d t \geq 0
$$

Thus, choosing $\delta \mathbf{v}^{n}$ as the gradient direction ensures a local improvement in the objective functional, $J_{A}$. At the higher and lower bounds on $\mathbf{v}^{n}$, we must make the appropriate weighting terms equal to zero to avoid leaving the allowable region.

By substituting the discrete governing equations (3.3) and the performance index (4.1) into (5.1), the required gradient for the OCP of polymer flooding is expressed as

$$
\delta \mathbf{v}_{i, j}^{n}=w\left(\lambda_{c i, j}^{n+1}-\Delta t^{n} \xi_{p}\right) Q_{\text {in } i, j^{\prime}}^{n} \quad(i, j) \in \kappa_{w}
$$

The computational algorithm of control iteration based on gradient direction is as follows.

Step 1. Make an initial guess for the control vector, $\mathbf{v}^{n}, n=0, \ldots, N-1$.

Step 2. Using stored current value of $\mathbf{v}^{n}, n=0, \ldots, N-1$, integrate the governing equations forward in time with known initial state conditions. Store the discrete states $\widetilde{\mathbf{u}}^{n+1}, n=$ $0, \ldots, N-1$.

Step 3. Calculate the profit functional with the results of the forward integration.

Step 4. Solve the adjoint equations using the stored discrete states to calculate the adjoint variables $\lambda^{n+1}, n=0, \ldots, N-1$, with (4.14) and (4.17). Compute and store $\delta \mathbf{v}^{n}$ as defined by (5.3).

Step 5. Using the evaluated $\delta \mathbf{v}^{n}$, an improved function is computed as

$$
\mathbf{v}_{\text {new }}^{n}=\mathbf{v}_{\text {old }}^{n}+\delta \mathbf{v}^{n}, \quad n=0,1, \ldots N-1 \text {, }
$$

where $\mathbf{0} \leq \mathbf{v}_{\text {new }}^{n} \leq \mathbf{v}_{\max }$. A single variable search strategy can be used to find the value of the positive weighting factor $w$ which maximizes the improvement in the performance functional using (5.4). 
Step 6. The optimization algorithm is stopped when the variation $\delta \mathbf{v}$ is too small to effectively change the performance measure, that is, when

$$
\left|J_{\text {new }}-J_{\text {old }}\right|<\varepsilon,
$$

where $\varepsilon$ is a small positive number.

A penalty function method is adopted to deal with the polymer injection amount constraint (4.4). For more information about that, we are able to use this method within gradient-based algorithm, see [19].

It is clear that one forward governing equations evaluation and one backward adjoint equations evaluation are required to calculate the gradients of the performance index, irrespective of the number of controls. The time required to solve the adjoint equations is of the same order of magnitude as the governing equations. Thus, with this process, a time equivalent to approximately two integrations is all that is required to calculate any number of gradients. This is why gradient-based algorithms can be very efficient and can potentially lead to huge time savings if the number of controls is large.

\section{New Algorithm for Adjoint Construction}

Despite the great efficiency of the gradient-based algorithm, a major drawback of the approach is that an adjoint code is required in order to apply the algorithm. The complexity of the adjoint equations is similar to that of the governing equations. A modified approach is proposed to construct the adjoint that makes it relatively easy to create the adjoint code. The approach is due to the properties of the full-implicit finite-difference code and the forms of the performance index used in the OCP model of polymer flooding.

The main coefficient terms of the adjoint equations given by (4.14) are the two Jacobians of the governing equations:

$$
\frac{\partial \mathbf{g}^{n}\left(\tilde{\mathbf{u}}^{n+1}, \tilde{\mathbf{u}}^{n}, \mathbf{v}^{n}\right)}{\partial \widetilde{\mathbf{u}}^{n}}, \quad \frac{\partial \mathbf{g}^{n-1}\left(\tilde{\mathbf{u}}^{n}, \tilde{\mathbf{u}}^{n-1}, \mathbf{v}^{n-1}\right)}{\partial \widetilde{\mathbf{u}}^{n}}
$$

The two terms are difficult to calculate because they are functions of the governing equations. During the forward integration of the governing equations, we solve (3.3) to determine $\widetilde{\mathbf{u}}^{n+1}$ at each time step. Since these equations are nonlinear with respect to $\widetilde{\mathbf{u}}^{n+1}$, the usual method to solve them is through the Newton-Raphson algorithm [20]:

$$
\begin{gathered}
\left.\frac{\partial \mathbf{g}^{n}}{\partial \widetilde{\mathbf{u}}^{n+1}}\right|_{\widetilde{\mathbf{u}}^{n+1, k}} \delta \widetilde{\mathbf{u}}^{n+1, k}=-\mathbf{g}^{n}\left(\widetilde{\mathbf{u}}^{n+1}, \widetilde{\mathbf{u}}^{n}, \mathbf{v}^{n}\right), \\
\widetilde{\mathbf{u}}^{n+1, k+1}=\widetilde{\mathbf{u}}^{n+1, k}+\delta \widetilde{\mathbf{u}}^{n+1, k},
\end{gathered}
$$


where $k$ is the iteration index of the Newton-Raphson algorithm at a given time step, $\widetilde{\mathbf{u}}^{n+1, k}$ is the state vector of the $k$ th iteration and $\left.\left(\partial \mathbf{g}^{n} / \partial \widetilde{\mathbf{u}}^{n+1}\right)\right|_{\widetilde{\mathbf{u}}^{n+1, k}}$ is the Jacobian of the $k$ th iteration which is calculated by

$$
\left.\frac{\partial \mathbf{g}^{n}}{\partial \widetilde{\mathbf{u}}^{n+1}}\right|_{\widetilde{\mathbf{u}}^{n+1, k}}=\frac{\partial \mathbf{F}^{n+1}}{\partial \widetilde{\mathbf{u}}^{n+1, k}}+\frac{\partial \mathbf{W}^{n+1}}{\partial \widetilde{\mathbf{u}}^{n+1, k}}-\frac{\partial \mathbf{A}^{n+1}}{\partial \widetilde{\mathbf{u}}^{n+1, k}}
$$

Equation (6.2) is linear with respect to $\delta \widetilde{\mathbf{u}}^{n+1, k}$. At the convergence of the algorithm, the Jacobian used in (6.2) is the same as the second Jacobian of (6.1).

Considering the full-implicit governing equations (3.3), the first Jacobian of (6.1) is given as

$$
\frac{\partial \mathbf{g}^{n}}{\partial \widetilde{\mathbf{u}}^{n}}=\frac{\partial \mathbf{A}^{n}\left(\widetilde{\mathbf{u}}^{n}\right)}{\partial \widetilde{\mathbf{u}}^{n}}
$$

The governing equations of the previous time step is expressed as

$$
\mathbf{g}^{n-1}=\mathbf{F}^{n}\left(\tilde{\mathbf{u}}^{n}\right)+\mathbf{W}^{n}\left(\tilde{\mathbf{u}}^{n}, \mathbf{v}^{n-1}\right)-\left[\mathbf{A}^{n}\left(\tilde{\mathbf{u}}^{n}\right)-\mathbf{A}^{n-1}\left(\tilde{\mathbf{u}}^{n-1}\right)\right]=\mathbf{0} .
$$

Then the second Jacobian for this time step is given by

$$
\frac{\partial \mathbf{g}^{n-1}}{\partial \widetilde{\mathbf{u}}^{n}}=\frac{\partial \mathbf{F}^{n-1}\left(\widetilde{\mathbf{u}}^{n}\right)}{\partial \widetilde{\mathbf{u}}^{n}}+\frac{\partial \mathbf{W}^{n-1}\left(\widetilde{\mathbf{u}}^{n}, \mathbf{v}^{n-1}\right)}{\partial \widetilde{\mathbf{u}}^{n}}-\frac{\partial \mathbf{A}^{n}\left(\widetilde{\mathbf{u}}^{n}\right)}{\partial \widetilde{\mathbf{u}}^{n}} .
$$

The last term of (6.7) is the same as the right side of (6.5). Thus, the first Jacobian of any given time step is calculated during the computation of the second Jacobian of the previous time step.

The rest terms of adjoint equations are the derivatives of the scalar performance index $J^{n-1}$ with respect to the state vector $\widetilde{\mathbf{u}}^{n}$. Due to the discrete form of performance index (4.1), the terms $\partial J^{n-1} / \partial \widetilde{\mathbf{u}}^{n}$ are directly functions of the source terms derivatives with respective to states $\partial \mathbf{W}^{n} / \partial \widetilde{\mathbf{u}}^{n}$. This is also calculated within the forward integration as seen from (6.4) and is easy to extract.

Thus, all the terms required for solving the adjoint equations can be calculated during the forward governing equations evaluation itself. The computational algorithm of control iteration based on gradient direction is modified as follows.

Step 1. Make an initial guess for the control vector, $\mathbf{v}^{n}, n=0, \ldots, N-1$.

Step 2. Using stored current value of $\mathbf{v}^{n}, n=0, \ldots, N-1$, integrate the governing equations forward in time with known initial state conditions. Store the discrete states $\widetilde{\mathbf{u}}^{n+1}$ and the coefficients involved in the adjoint equations $\partial \mathbf{g}^{n} / \partial \widetilde{\mathbf{u}}^{n}, \partial \mathbf{g}^{n} / \partial \widetilde{\mathbf{u}}^{n+1}, \partial \mathbf{W}^{n+1} / \partial \widetilde{\mathbf{u}}^{n+1}, n=$ $0, \ldots, N-1$.

Step 3. Calculate the profit functional with the results of the forward integration. 


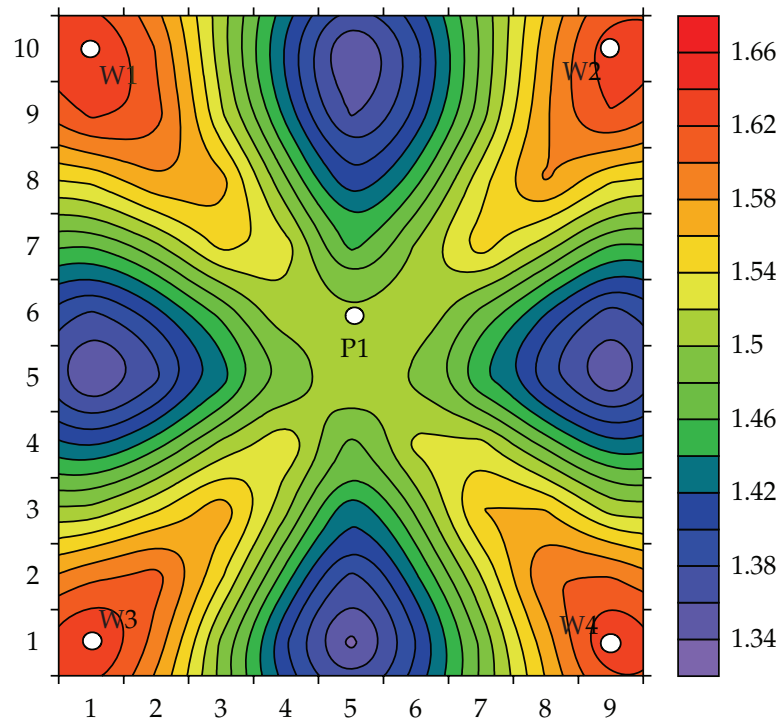

Figure 2: Permeability $\left(\mu \mathrm{m}^{2}\right)$ distribution.

Step 4. Solve the adjoint equations using the stored Jacobians and source terms derivatives in Step 2 to calculate the adjoint variables $\lambda^{n+1}, n=0, \ldots, N-1$, with (4.14) and (4.17). Compute and store $\delta \mathbf{v}^{n}$ as defined by (5.3).

Step 5. Using the evaluated $\delta \mathbf{v}^{n}$, an improved function is computed as (5.4).

Step 6 . The optimization algorithm is stopped when the variation $\delta \mathbf{v}$ is too small to effectively change the performance measure.

It should be noted that mathematically there is no change in the algorithm, but the adjoint equations become much simpler to code. Furthermore, the adjoint equations code can be fully consistent with the governing equations if the polymer flooding model is changed. For example, some terms reflecting new physical characteristics are added to (3.3). This is because the coefficient terms in adjoint equations are taken directly from the governing equations in the proposed method.

\section{Case Study}

In this section, we present a numerical example of optimal control for polymer flooding done with the proposed implicit discrete maximum principle method.

The two-phase flow of oil and water in a heterogeneous two-dimensional reservoir is considered. The reservoir covers an area of $421.02 \times 443.8 \mathrm{~m}^{2}$ and has a thickness of $5 \mathrm{~m}$ and is discretized into $90(9 \times 10 \times 1)$ grid blocks. The production model is a five-spot pattern, with one production well P1 located at the center of the reservoir $(5,6)$ and four injection wells W1-W4 placed at the four corners $(1,10),(9,10),(1,1)$, and $(9,1)$ as shown in the permeability distribution map of Figure 2. Polymer is injected when the fractional flow of water for the production well comes to $97 \%$ after water flooding. The time domain of polymer injection is $0-1440$ days and the polymer flooding project life is $t_{f}=5500$ (days). 


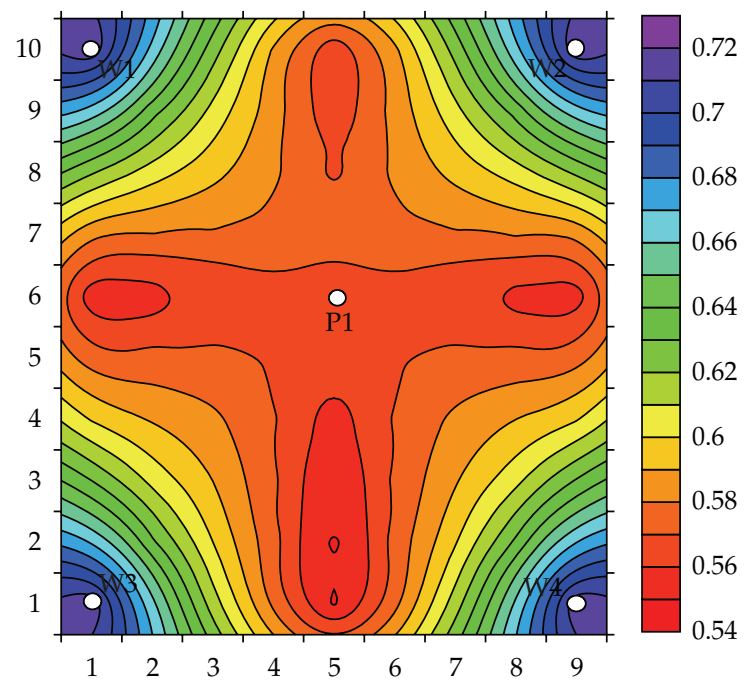

Figure 3: Initial water saturation contour map.

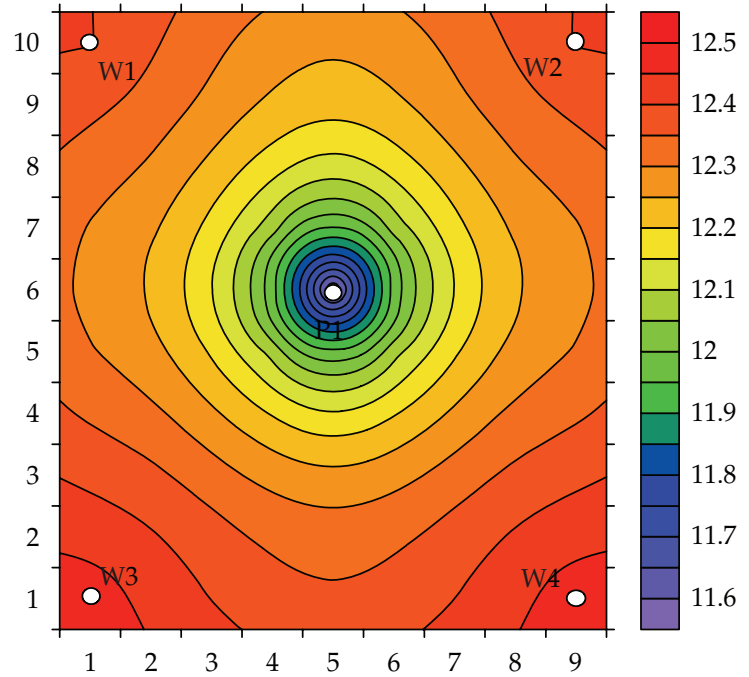

Figure 4: Initial reservoir pressure $(\mathrm{MPa})$ contour map.

Figures 3 and 4 show the contour maps of the initial water saturation $S_{w}^{0}$ and the initial reservoir pressure $p^{0}$, respectively. The initial polymer concentration is $c_{p}^{0}=0(\mathrm{~g} / \mathrm{L})$. In the performance index calculation, we use the price of oil $\xi_{o}=0.0503\left(10^{4} \$ / \mathrm{m}^{3}\right)[80(\$ / \mathrm{bbl})]$, and the cost of polymer $\xi_{p}=2.5 \times 10^{-4}\left(10^{4} \$ / \mathrm{kg}\right)$. The fluid rate of the production well is $Q_{\text {out }}=60 \mathrm{~m}^{3} /$ day, and the fluid rate of every injection well is $Q_{\text {in }}=15 \mathrm{~m}^{3} /$ day. The PDEs are solved by full-implicit finite-difference method with step size 10 days. For the constraint (2.15), the maximum injection polymer concentration is $c_{\max }=2.2(\mathrm{~g} / \mathrm{L})$. The parameters of the reservoir description and the fluid data are shown in Tables 1 and 2, respectively. 


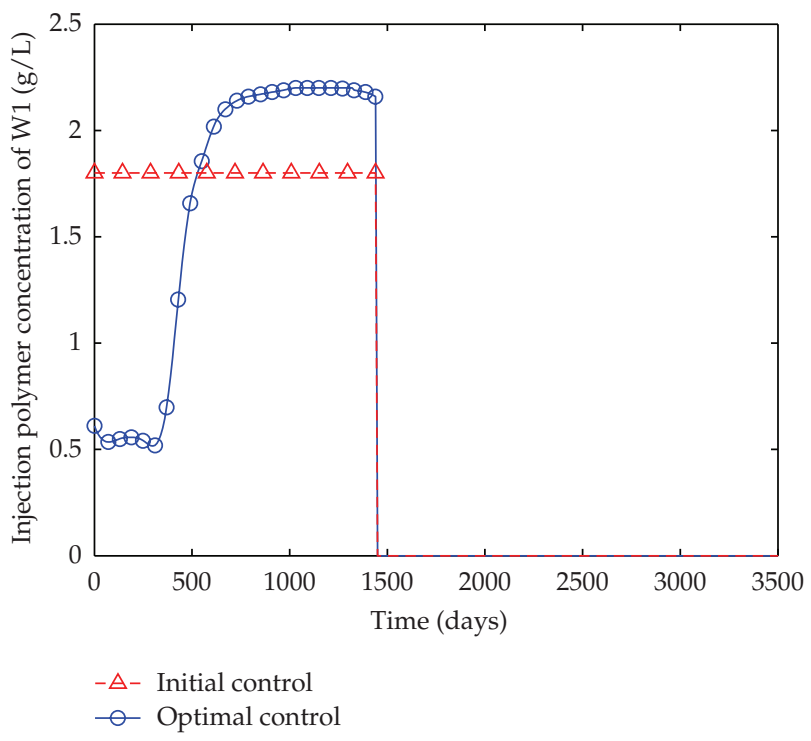

Figure 5: Injection polymer concentration of well W1.

The polymer injection strategies obtained by the conventional engineering judgment method (trial and error) are the same $1.8(\mathrm{~g} / \mathrm{L})$ for all injection wells. The performance index is $J=\$ 1.592 \times 10^{7}$ with oil production $32429 \mathrm{~m}^{3}$ and polymer injection $155520 \mathrm{~kg}$. For comparison, the results obtained by engineering judgment method are considered as the initial control strategies of the proposed implicit discrete maximum principle method. The maximum polymer injection amount in constraint $(2.14)$ is $m_{p \max }=155520(\mathrm{~kg})$. A backtracking search strategy [19] is used to find the appropriate weighting term $w$ and the stopping criterion is chosen as $\varepsilon=1 \times 10^{-5}$. By using the proposed algorithm, we obtain a cumulative oil of $33045 \mathrm{~m}^{3}$ and a cumulative polymer of $155520.01 \mathrm{~kg}$ yielding the profit of $J^{*}=\$ 1.623 \times 10^{7}$ over the polymer flooding project life of the reservoir. The results show an increase in performance index of $\$ 3.1 \times 10^{5}$. Figures $5,6,7$, and 8 show the optimal control policies of the injection wells W1-W4. As a result, the optimal injection polymer concentration profiles of W1, W2 are significantly different from those of W3, W4. It is mainly due to the differences of the well positions and the distance to the production well, as well as the reservoir heterogeneity and the uniform initial water saturation distribution. Figures 9 and 10 show the fractional flow of water in production well and the cumulative oil production curves of the two methods, respectively. It is obvious that the fractional flow of water obtained by implicit discrete maximum principle method is lower than that by engineering judgment. Therefore, with the same cumulative polymer injection, the proposed method gets more oil production and higher recovery ratio.

\section{Conclusion}

In this work, a new optimal control model of DPS is established for the dynamic injection strategies making of polymer flooding. Necessary conditions of this OCP are obtained by 


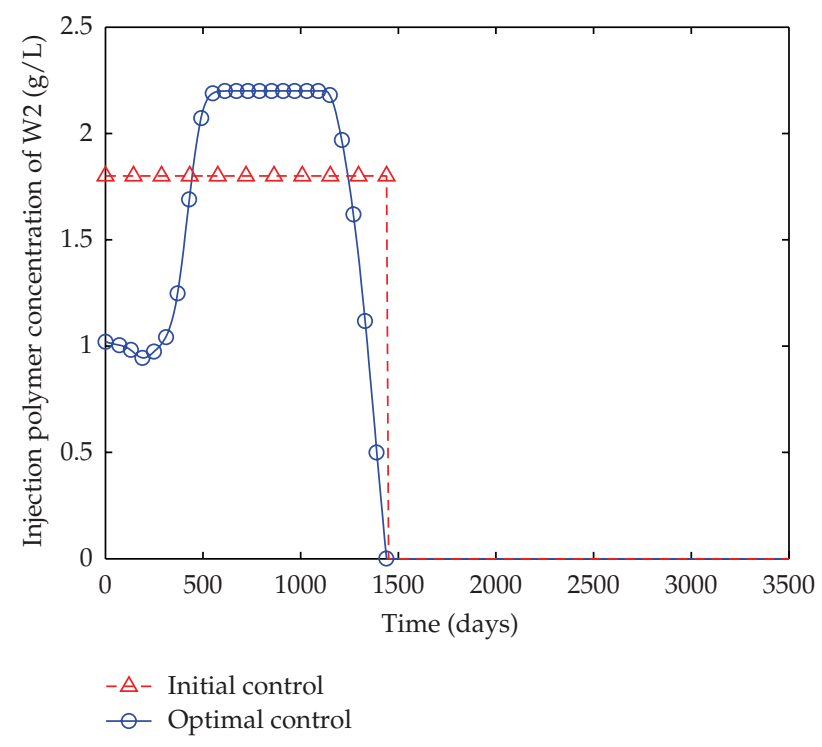

Figure 6: Injection polymer concentration of well W2.

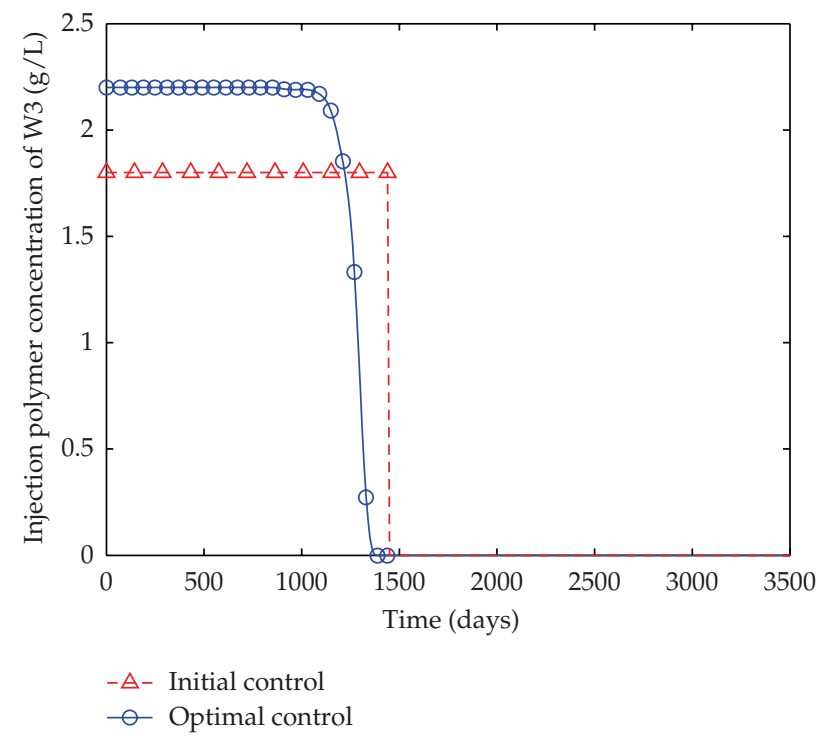

Figure 7: Injection polymer concentration of well W3.

using the calculus of variations and Pontryagin's discrete maximum principle. A gradientbased iterative computational algorithm with new adjoint construction is proposed for the determination of optimal injection strategies. It is shown that the modified approach makes it relatively easy to code the adjoint equations as compared to the standard approach. The optimal control model of polymer flooding and the proposed method are used for a reservoir example and the optimum injection concentration profiles for each well are offered. The results show that the profit is enhanced by the proposed method. Meanwhile, more oil 


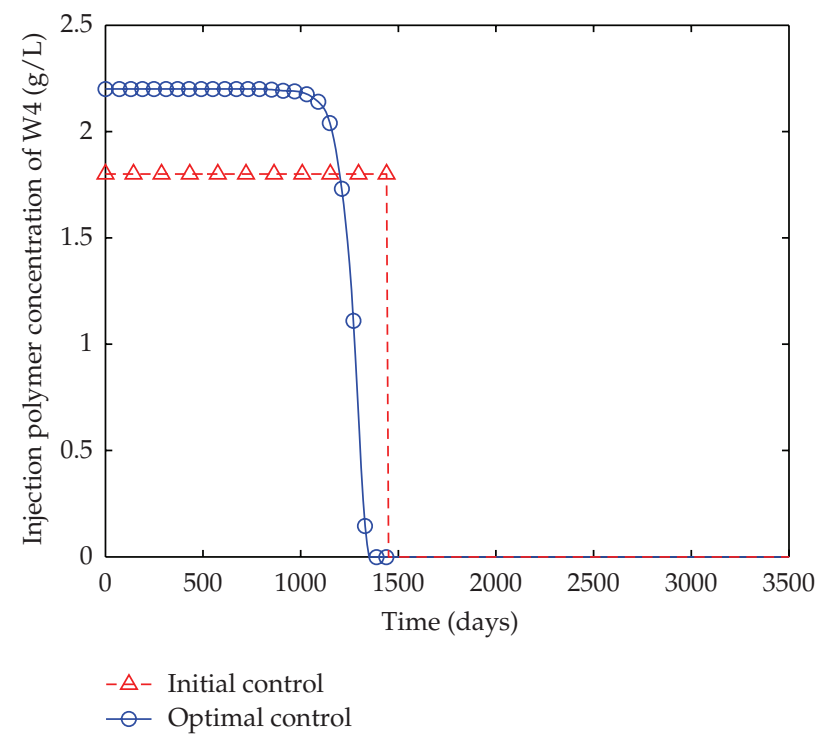

Figure 8: Injection polymer concentration of well W4.

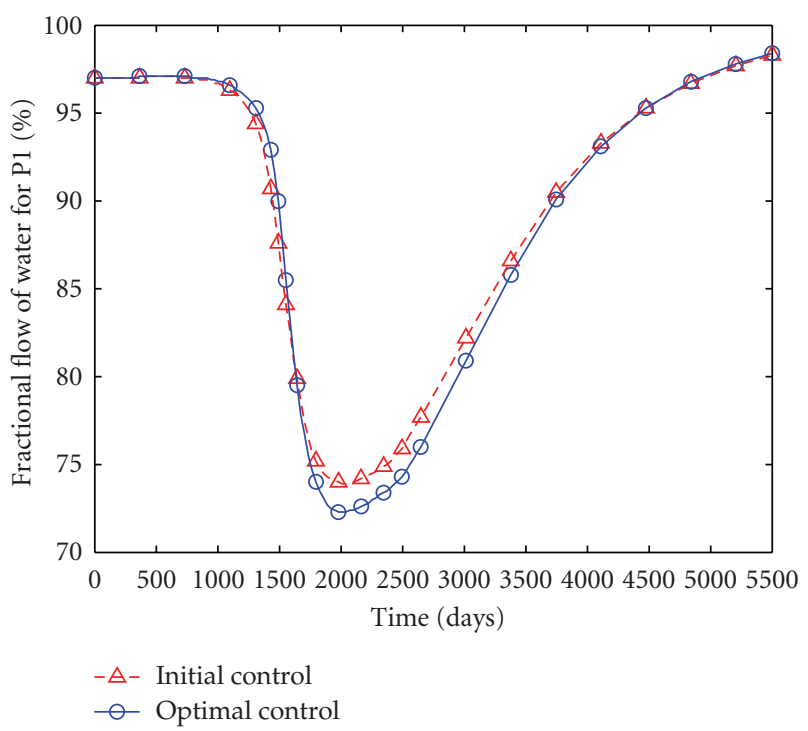

Figure 9: Fractional flow of water for the production well P1.

production and higher recovery ratio are obtained. And the injection strategies chosen by engineering judgment are the same for all the wells, whereas the optimal control policies by the proposed method are different from each other as a result of the reservoir heterogeneity and the uniform initial conditions.

In conclusion, given the properties of an oil reservoir and the properties of a polymer solution, optimal polymer flooding injection strategies to maximize profit can be designed by 


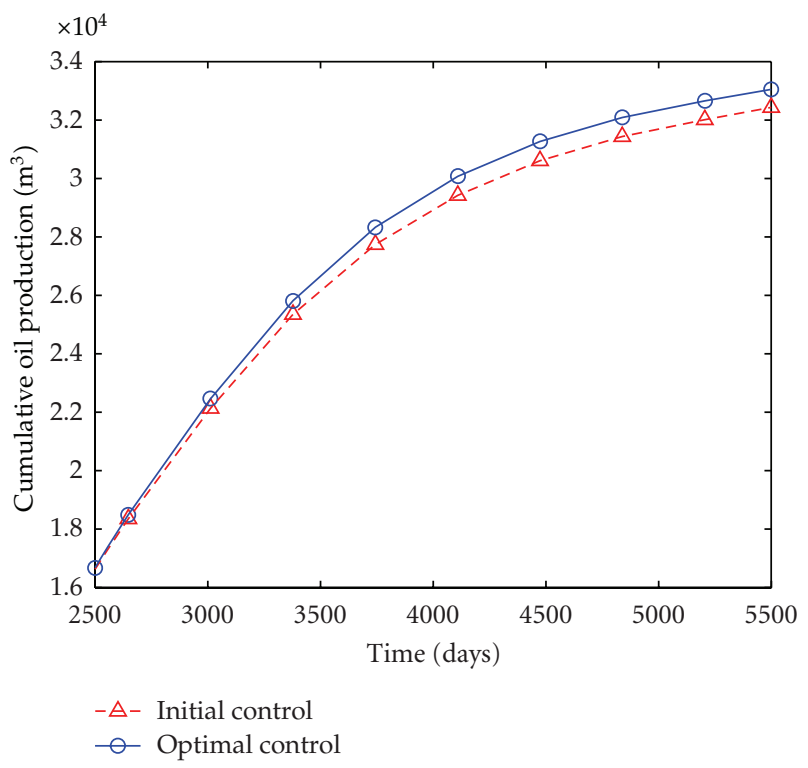

Figure 10: Cumulative oil production.

Table 1: Parameters of reservoir description used in the example.

\begin{tabular}{lc}
\hline Parameters & Values \\
\hline Number of production well, $N_{p}$ & 1 \\
Number of injection wells, $N_{w}$ & 4 \\
Thickness of the reservoir bed, $h(\mathrm{~m})$ & 5 \\
Reference pressure, $p_{r}(\mathrm{MPa})$ & 12 \\
Porosity under the condition of the reference pressure, $\phi_{r}$ & 0.31 \\
Rock density, $\rho_{r}\left(\mathrm{~kg} / \mathrm{m}^{3}\right)$ & 2000 \\
Rock compressibility factor, $C_{R}(1 / \mathrm{MPa})$ & $9.38 \times 10^{-6}$ \\
Irreducible water saturation, $S_{o r}$ & 0.25 \\
Residual oil saturation, $S_{w c}$ & 0.22 \\
Oil relative permeability at the irreducible water saturation, $k_{r w r o}$ & 0.5228 \\
Water relative permeability at the residual oil saturation, $k_{r o c w}$ & 0.9 \\
Index of oil relative permeability curve, $n_{o}$ & 4.287 \\
Index of water relative permeability curve, $n_{w}$ & 2.3447 \\
\hline
\end{tabular}

using implicit discrete maximum principle. The approach used is a powerful tool that can aid significantly in the development of operational strategies for EOR processes.

\section{Acknowledgments}

This work was supported by the Natural Science Foundation of China (60974039), the Natural Science Foundation of Shandong Province of China (ZR2011FM002), the Fundamental Research Funds for the Central Universities (27R1105018A), and the Postgraduate Innovation Funds of China University of Petroleum (CX-1245). The authors would like to express their 
Table 2: Fluid data used in the example.

\begin{tabular}{lc}
\hline Parameters & Values \\
\hline Oil viscosity, $\mu_{o}(\mathrm{mPa} \cdot \mathrm{s})$ & 50 \\
Compressibility factors of oil, $C_{o}(1 / \mathrm{MPa})$ & $5 \times 10^{-6}$ \\
Oil volume factor under the condition of the reference pressure, $B_{\text {or }}$ & 1 \\
Aqueous phase viscosity with no polymer, $\mu_{w}(\mathrm{mPa} \cdot \mathrm{s})$ & 0.458 \\
Compressibility factors of water, $C_{w}(1 / \mathrm{MPa})$ & $4.6 \times 10^{-6}$ \\
Water volume factor under the condition of the reference pressure, $B_{w r}$ & 1 \\
Polymer absorption parameter, $a\left(\mathrm{~cm}^{3} / \mathrm{g}\right)$ & 0.03 \\
Polymer absorption parameter, $b(\mathrm{~L} / \mathrm{g})$ & 3.8 \\
Diffusion coefficient, $D\left(\mathrm{~m}^{2} / \mathrm{s}\right)$ & $1 \times 10^{-5}$ \\
Effective pore volume coefficient, $f_{a}$ & 1 \\
Permeability reduction parameter, $R_{k \text { max }}$ & 1.15 \\
Permeability reduction parameter, $b_{r k}$ & 1.2 \\
Viscosity parameter, $a p_{1}$ & 15.426 \\
Viscosity parameter, $a p_{2}$ & 0.4228 \\
Viscosity parameter, $a p_{3}$ & 0.2749 \\
\hline
\end{tabular}

great appreciation to the editors and anonymous referees for their helpful and constructive comments and suggestions, which have helped to improve this paper.

\section{References}

[1] Y. Qing, D. Caili, W. Yefei, T. Engao, Y. Guang, and Z. Fulin, “A study on mass concentration determination and property variations of produced polyacrylamide in polymer flooding," Petroleum Science and Technology, vol. 29, no. 3, pp. 227-235, 2011.

[2] B. K. Maitin, "Performance analysis of several polyacrylamide floods in North German oil fields," in Proceedings of the Eighth Symposium on Enhanced Oil Recovery, pp. 159-165, April 1992.

[3] M. Kumar, V. Hoang, C. Satik, and D. Rojas, "High-mobility-ratio-waterflood performance prediction: challenges and new insights," SPE Reservoir Evaluation and Engineering, vol. 11, no. 1, pp. 186-196, 2008.

[4] Q. Yu, H. Jiang, and C. Zhao, "Study of interfacial tension between oil and surfactant polymer flooding," Petroleum Science and Technology, vol. 28, no. 18, pp. 1846-1854, 2010.

[5] H. Jiang, Q. Yu, and Z. Yi, "The influence of the combination of polymer and polymer-surfactant flooding on recovery," Petroleum Science and Technology, vol. 29, no. 5, pp. 514-521, 2011.

[6] W. F. Ramirez, Z. Fathi, and J. L. Cagnol, “Optimal injection policies for enhanced oil recovery: part 1theory and computational strategies," Society of Petroleum Engineers journal, vol. 24, no. 3, pp. 328-332, 1984.

[7] Z. Fathi and W. F. Ramirez, "Use of optimal control theory for computing optimal injection policies for enhanced oil recovery," Automatica, vol. 22, no. 1, pp. 33-42, 1986.

[8] W. Liu, W. F. Ramirez, and Y. F. Qi, "Optimal control of steamflooding," SPE Advanced Technology Series, vol. 1, no. 2, pp. 73-82, 1993.

[9] J. Ye, Y. Qi, and Y. Fang, "Application of optimal control theory to making gas-cycling decision of condensate reservoir," Chinese Journal of Computational Physics, vol. 15, no. 1, pp. 71-76, 1998.

[10] D. R. Brouwer and J. D. Jansen, "Dynamic optimization of water flooding with smart wells using optimal control theory," in Proceedings of the 13th SPE European Petroleum Conference, pp. 391-v, Aberdeen, Scotland, October 2002.

[11] P. Sarma, K. Aziz, and L. J. Durlofsky, "Implementation of adjoint solution for optimal control of smart wells," in Proceedings of the SPE Reservoir Simulation Symposium, pp. 1-17, The Woodlands, Tex, USA, February 2005.

[12] K. Zhang, J. Yao, L. Zhang, and Y. Li, "Dynamic real-time optimization of reservoir production," Journal of Computers, vol. 6, no. 3, pp. 610-617, 2011. 
[13] J. D. Jansen, "Adjoint-based optimization of multi-phase flow through porous media-a review," Computers and Fluids, vol. 46, no. 1, pp. 40-51, 2011.

[14] L. L. Guo, S. R. Li, Y. B. Zhang, and Y. Lei, "Solution of optimal control of polymer flooding based on parallelization of iterative dynamic programming," Zhongguo Shiyou Daxue Xuebao, vol. 33, no. 3, pp. 167-174, 2009.

[15] Y. Lei, S. Li, X. Zhang, Q. Zhang, and L. Guo, "Optimal control of polymer flooding based on mixedinteger iterative dynamic programming," International Journal of Control, vol. 84, no. 11, pp. 1903-1914, 2011.

[16] S. Li, Y. Lei, X. Zhang, and Q. Zhang, "Optimal control solving of polymer flooding based on a hybrid genetic algorithm," in Proceedings of the 29th Chinese Control Conference (CCC '10), pp. 5194 5198, Beijing, China, July 2010.

[17] Y. Lei, S. Li, and X. Zhang, "Optimal control solving of polymer flooding based on real-coded genetic algorithm," in Proceedings of the 8th World Congress on Intelligent Control and Automation (WCICA '10), pp. 5111-5114, Jinan, China, July 2010.

[18] Y. Lei, S. Li, Q. Zhang, X. Zhang, and L. Guo, “A hybrid genetic algorithm for optimal control solving of polymer flooding," in $v$ International Conference on Intelligent Computation Technology and Automation (ICICTA '10), pp. 122-125, Changsha, China, May 2010.

[19] J. Nocedal and S. J. Wright, Numerical Optimization, Springer Series in Operations Research and Financial Engineering, Springer, New York, NY, USA, 2nd edition, 2006.

[20] K. Aziz and A. Settari, Fundamentals of Reservoir Simulation, Elsevier Applied Science, New York, NY, USA, 1986. 


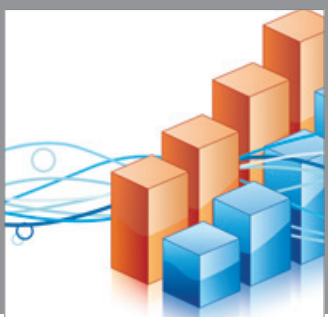

Advances in

Operations Research

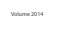

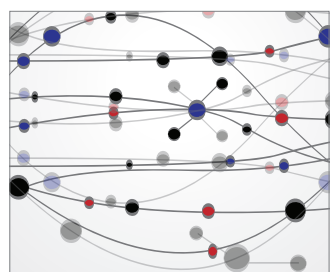

\section{The Scientific} World Journal
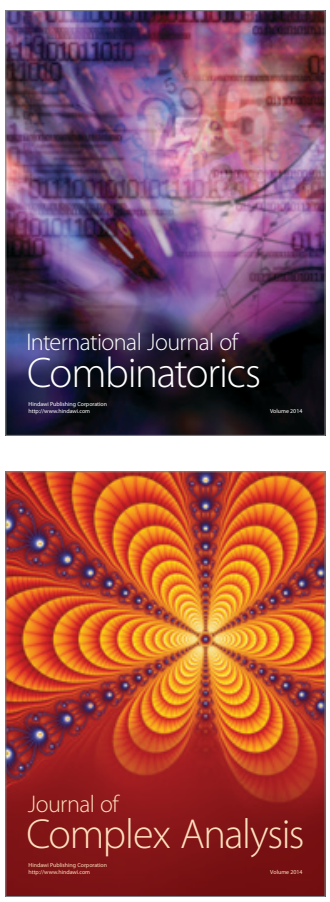

International Journal of

Mathematics and

Mathematical

Sciences
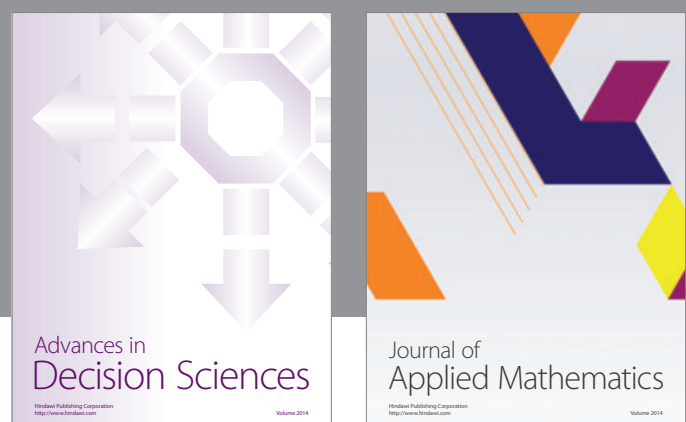

Journal of

Applied Mathematics
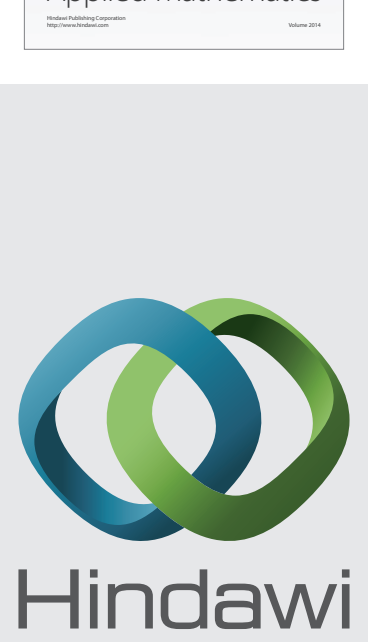

Submit your manuscripts at http://www.hindawi.com
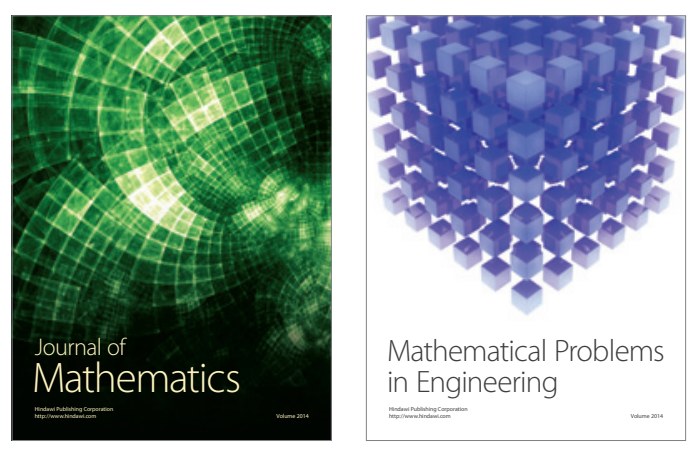

Mathematical Problems in Engineering
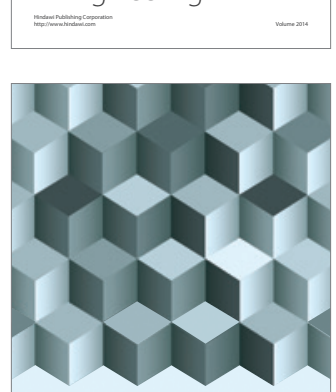

Journal of

Function Spaces
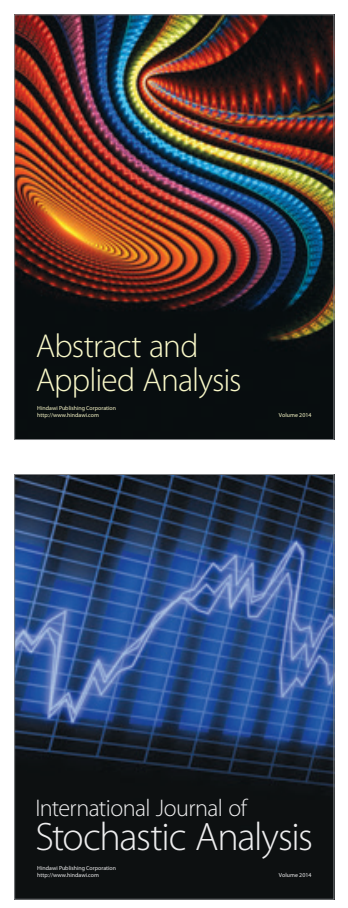

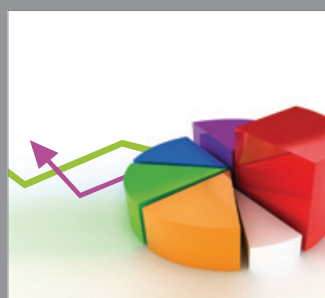

ournal of

Probability and Statistics

Promensencen
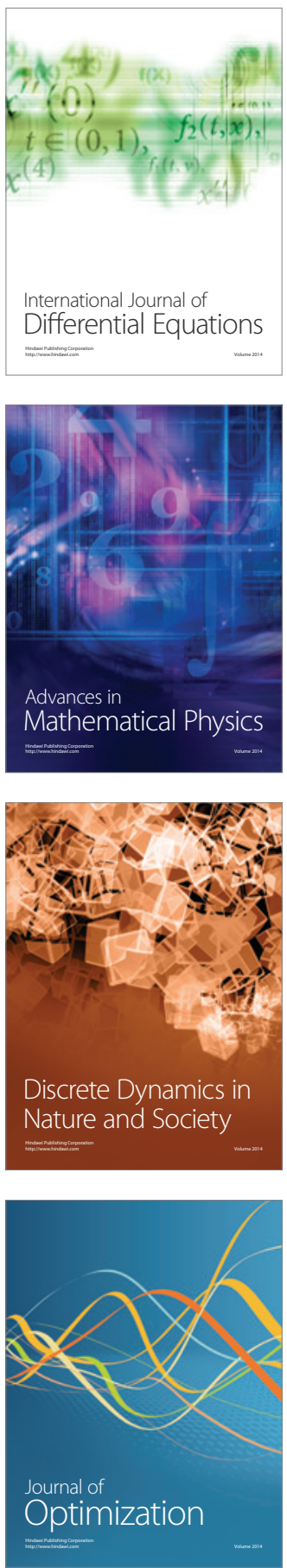